\title{
ARTIFICIAL NEURAL NET BASED NOISE CANCELLOR
}

\author{
P.K. Dash, S. Saha, P.K. Nanda, \\ Electrical Engineering Department, Regional Engineering College, \\ Rourkela 769008 \\ H.P. Khincha, \\ Electrical Engineering Department, \\ Indian Institute of Science, \\ Bangalore 560012
}

\begin{abstract}
This paper presents a new method for noise cancellation with an Artificial Neural Network. The network used is a feedforward one with three layers. The backpropagation and stastical Cauchy"s learning lgorithms are employed for adaptation of the internal parameters of the network. The constrained tangent hyperbolic function is used to activate the neurons and to provide the desired non-1inearity. Promising simulation results for noise cancellation intensify the validlty of superseding the proposed scheme for many existing techniques. To demonstrate the effectiveness. the proposed method is applied to different input conditions with varying SNRs. With incomplete signal samples the net is found to produce output having a striking resemblance with that of the desired ones. A performance comparision of the two algorithms is presented in the paper for better appraisal.
\end{abstract}

\section{INTRODUCII ON}

The usual method of estimating a signal corrupted by additive noise is to pass it through 3 filter that tends to suppress the noise while leaving the signal relatively unchanged. The filters for !he above purpose may be fixed or adaptive, where the latter one has the ability to adjust their own parameters automatically and their design requires little or no a prior 1 knowledge of signal or noise characterstics. In eircumstances where adaptive noise cancellation is applicable (1) levels of noise rejection are attainable that would be difficult or impossible to achieve by direct filtering. A good number of adaptive algorithms have been reported in the literature over the past few decades to update the internal parameters with a view to obtain high fidelity of the output signal in many complex environments. However the major focus of research over the past few years is to develop robust algorithms for adaptive systems.

However the past few years witnessed the flinching of the researchers to the field of Artificial Neural NetCANNS. The potential features of the ANN motivated to pursue research in the field with much accelerated vigour with a hope of achieving human like performance in the fields of speech and image recognition $[\hat{2}, \hat{3}]$. In addition to the above features neural networks can provide solution to optimization, handwritten diglt-recognition, radar target detection. text-to-speech synthesis and computational models of brain functions. Adaptive non-parametric neural networks designed for pattern classification work well for many real world problems \{4\}. Neural net models are spelited by the net topology.. node characteristics. and training or learning rules. Both the design procedures and the training rules for supervised and unsupervised training are the topic of much current research $[5,6,7]$. The application of feedforward multi layered neural net models to spectral estimation have been reported in few research papers [8,9]. The application of neural net to the field of noise cancellation is hardly found in the 1iterature.

In this paper a Neural Net based Noise Canceller is proposed. A three layered feedforward network is employed for noise cancellation. The backpropagation algorithm and Statistical Cauchy's algorithm with Boltzmann's probability distribution is employed for updating the internal parameters i.e. weight of the net. Sinee both positive and negative time domain output sampler are of equal importance. the tangent hyperbolic function. constrained to operate in non-linear zone. is used to activate the neurons and to provide the dosirod non-inserity. The tangent hyperbolic function saturates at unity for small magnitudes of both positive and negative values. hence the maximum value of tho activation function $1=$ sutably adjuatod and tho output of $a$ neuron is zone Interforence and additive uncorrelated white noise bears a striking resemblance with that of the desired ones.

The fundamental signal components primarily of power frquency and its corresponding third and fifth harmonic components are succorstully extracted from the corrupted signal with $10 \mathrm{w}$ SNRs. The net is also exposed to many never seen data other than the trained ones and the nolse cancellation feature is commendable. The net possesses potentiality to extract the desired components even with 50\% suppressed input data. both with predominant $d . c$ and with appreciable harmonic strength, without signal degradation. Sufficient computer simulation results are presented for a wide yar lety of cases. 


\section{ARTIFICIAL. NEURAL NETWORK}

Interest in artificial neural networks has grown rapidly over the past few years. This insurgence of interest fired by both theoritleal and application successes motivated to make machines that learn and rememer in ways that boar a striking resemblance to human mental processes and asslgned a new significant meaning to Artificial Intelligence. In 1950 s and $1000 \mathrm{~s}$ a group of researohers combined the biological and psychological insights to produce the first artificial neural network model. Due to limitation in representational capability of the single neuron and single layer neurons, multilayered perceptrons were developed which invoked the development of backpropagation training algortthm by Parker [10].

Development of detailed mathematical models began more than four decades ago with the work of McCulloen and Pitts. Hebb. Rasenblatt. Widrow and others. More recent work by Hoffield. Rumelhart Mecelland [11]. Sejnowski, Feldman. Grossberg. Widrow ( 12,13 ) and others led to a new insurgence in the field. Over the past few years the research about neural net has been persued with accelerated vigours, sires the advent of new net topologies, robust algorithms. analog VLS implementation and the concept of parallel processing. These models are specified by the net topology, node characteristics and training or learning rules. These rules specify an initial set of weights and indicate how weights should be adapted during use to improve the performance. Adaptation or learning is a major focus of neural net research. The ability to adapt and continue learning is essential in areas such as speech recognition where training data is limited and new talkers. new words. new phrases and new environments are continously encountered.

However the millilayered ANN with backpropagation $2190 \mathrm{l} 1 \mathrm{th}$ is most sommondy used for many practical problems. A typical three layered network shown in Fig. (1) is trained with supervision. The three layered network has input layer. hidden layer and output layer consisting of multiple non-linear neurons associated with suitable activation function. Each input pattern to the net feeds up through the hidden layers upto the output layer. Each neuron forms the weighted sum of the inputs and pass it through the hidden layers upto the output layer. Each neuron forms the weighted sum of the inputs and pass it through the non-linear activation function to produce a output which serves as the input to the next layer i.e. output layer. The net is trained to minimize the objective function which is defined as the half of the sum of the squares of the differences between the predicted ones and the corresponding desired component. Once the network is trained it has the capability of producing output very close to the desired one with input patterns other than the trained ones thus establishing tho validity for real tine implementation.

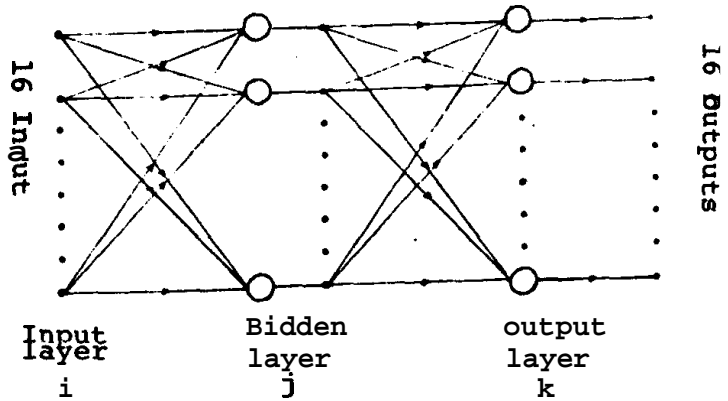

Fig. 1, A typical 3 layered feedforward Artificial Neural Network.

\section{TRAINING ALGOEI THNS}

The net trained to justify the noise cancellation capability $1 \mathrm{~s}$ shown in Fig. (1). The subscripts $1, \mathrm{j}$ and $\mathrm{k}$ refer to the input. hidden and output layer respectively and the subscripts $n$. $m$ and 1 sorrospond to any unit ir input. hidenr arud output layer. The net is trained with supervision by the required input and target vectors.

The two algorithms employed for training are backpropagation and statistical Cauchy's algorithm which are presented below.

Backpropagation Algorithm:

For each time step the following steps are to be axencutad.

Step-1: Initilisation of weights. Set the the weights of both the layers to small random values

stop- tho input voctor $x_{0} \ldots \ldots x_{N-1}$ and the target vector $d_{0}, \ldots, d_{N-1}$ are formed and applied to the net.

Steo-3: Calculate the actual outputs of both tho layers

$$
\begin{aligned}
& \hat{y}=s<\lambda \cdot \underline{x} \cdot(w) 3 \\
& \hat{y}=\text { Estimated Output }
\end{aligned}
$$$$
\lambda=\text { Weighting factor to }
$$
constrain the activation function in the non-1inear zone.

$$
[\psi]=\text { Weight matrix between any }
$$
two layers.

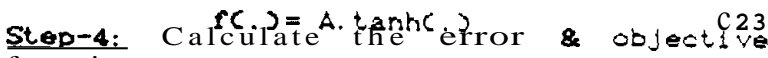
function.

$$
\begin{aligned}
& \cdot=d-\hat{y} \\
& E=(1 / 2) \sum_{p}\left(d_{k}-\hat{\imath}_{k} z\right. \\
& E_{p}^{P}=\text { objective function }
\end{aligned}
$$

Step-5: Adapt the weight.

$$
\text { (w] }(t+1)=[w] i f(t)+\eta \cdot \delta x \text { (5) }
$$
where $i d w\}$ (t) lsijtho wigb住j from $1 \mathrm{~J}$

hidden node 1 or from an input to node $j$ at $t i$ mo $t, \underline{x}_{j} i \leq$ eithor tho output of node or is an input, $n$ is a convergence co-efficient varied from 0.1 to $1.0 . \delta_{j}$ is an error term for node $j$. If node $j 1 s$ an output node thes

$$
\left.\sigma_{j}=\left(A-\hat{y}_{j}^{2} / A\right) C d_{j}-\hat{y}_{j}\right)
$$


when $d_{j}$ is the desired output of node $j$ and $y_{j}$ is the actual output.

If node $j$ is an internal hidden then

$$
w \sigma_{j}=\mathrm{CA}-\mathbf{x}_{j}^{\bar{\sigma}_{j} / A} \sum \delta_{k}[w] j k
$$

where $k$ is over all nodes to the right of node $j$.

The steps from 1 to $s$ are repeated till the objoct 1 ve function is minimized.

Cauchy's Algorithm:

The following stops are adopted for training the network.

Step-1: A variable $T$ that represents an initial artificial temperature is choosen. Usually $T$ is initialized to a large value. Also another variable. $t=$ artificial time analogous to a stop, is initialised. Usually $t$ is set to a small value. Here $T=8898,0, t=2,0$.

Step 2: A set of input is applied to the network, tho corresponding output and the objective function is evaluated.

Step-3: The weights are changed by a random value which is given as

$x=\operatorname{ex}(t) \cdot \tan [P(x)]$

(8)

where $\rho=$ the learning rate co-efficient

$x=$ the weight change

$P(X)=$ a radom number selected from 2 uniform distribution over the open interval -n\& to $\pi / z$.

Step-4: If the objective function is Improvedreduced, retain the weight change.

$$
E_{p}=1 / 2 \Sigma\left(d_{j}-\hat{y}_{j}\right)^{2}
$$

Step-5: If the weight change results in an increase in the objective function. calculate the probability of accepting that change from the Boltzmann's distribution as fO1lows.

$$
\text { PCo) }=\exp (-c / k T)
$$

where $860 \mathrm{c}=\mathrm{the}$ probability of a change of c in the oblective function.

$\mathrm{k}=\mathrm{a}$ constaric analogous to Boltzmann's constant that must Here $k=10^{-17}$ is selected.

$\mathrm{T}=$ the artificial temperature

The above steps are repeated until the objective function is minimized.

\section{RESULTS \& DISCUSSION}

In this research the network is trained independently for each case namely. fundamental, 3rd Harmonic \& $5 \mathrm{th}$ Harmonic etc. The signal generated is goverened by

$y(t)=\sum \sin (k \omega t)+v(t)$

where $k=1,3,5,7, \ldots n$ \& $v(t)$ is zero mean white noise. The objective functions for the fundamental, $3 \mathrm{rd}$ harmonic and $5 \mathrm{th}$ harmonic are presented in Figs. $2,8,7 \& 11$. Fig. 6 shows the local minima trapping with backpropagation algorithm for third harmonic which is alleviated using Cauchy's algorithm although it is slower in convergence as depicted in' Fig. 7. The input with varying SNRs and the corresponding outputs bearing a close resemblence to that of the desired ones, are represented in Figs. 4.9.10 \& 14 Fig. 3,6
\& 12 are the target waveforms for fundamental. $3 \mathrm{rd}$ harmonic and $5 \mathrm{th}$ harmonic respectively. Fig. $5 \& 13$ exhibit the inadequate input samples and the predicted outputs which ar upto the marks and hence it is elaimed that Neural $\mathrm{Net}$ based noise canceller is superior to the oujeting mothods.

\section{CONCLUSION}

Exhaustive computer simulation results establish tho validity to opt for the proposed scherer the existing digital filters. The performance in extracting the signal components from inadequate corrupted data excel over the existing techiniquer.

The local minima trapping effect of the objective function is circumvented by swithing over to cauchy's algorithm. The combined backpropagation and Cauchy's algorithm is being studied to overcome local minima trapping and to achieve faster convergence. The net is found to be tault tolerant and real time implementation of the proposed scheme seems to be more promising. Development and implementation of the generallsed filter is in Progress by employing both adaptive Pattern Racognttion concept and robust training algorithm.

\section{ACKNOMLDGEMENT}

The authors acknowledge with thanks the Ministry of Human Resource Development, covt. of India for providing necessary funds.

[1] B.Widrow t al, "Adaptive noise cancelling principles and applications". Proc. IEE , vol.83, pp. 1892-1716, Dec. 1075 .

bej R.P.Lippmann, "An Introduction to computing with Neural Nets", IEEE ASS Mag. Yol, 4(2), pp 4-22, April, 1987.

[3] A. Haibel. H. Sawai and $k$. shikand. Modularity and ssaling in Large phonemic Neural Network". IEEE Trans. on ASSP Vol. 37. No, 12, Doc. 1989.

[4] R.P.Lippmann, "Patzern classification using neural network", IEEE Communication Magazine PP 27-62. Nov. 1989.

[S] M.R. Azimi Sadjadi.S.Citren and Sassar Sheedrash. "Supervised Learning Process of Multi-Layer Perceptron Neural Network using Fast Least Squares", IGASS, April 3-G, 1990. New Mexico.U. S. A.

[8] P. Burrascano and P. Lucci, "A Learning rule dominating Local minima in muld layer perceptons" ICASSP April 3-6 $1990 \mathrm{New}$ Hexico U. S. A.

C 71 S.Y. Kung and K. I. Di amantras, "A Neural Network Learning algorithm for adaptative Principal Componenet Extraction (APEX)" I CASSP April 3-8, 1000. New Mexico U.S.A. C81 Chiz-Jio Wang \& Mark A. Wickert and C.H.H1e, "Three layer Neural Network for spectral estimation" ICASSP, April 3-6. 1990, New Mexico, USA.

(9) P. K. Dash, P. K. Nanda and S. Saha, " Spectral estimation listing Attificial Neural Network". International Conference on SIGNALS \& SYSTEMS, Dec, 10-12, Tirupati. India.

C101 Phi11ip D. Wasserman, "Neural Computing. Thoory and Practice" Van Nostrand \& Rolnhold 100

C 11] D. E. Rumeinart and J.L. HeCelland.

"Parallel Distributed.Processing". Vol. 1 \&

2 Cambridge. MA. MI T Press 1980. 
C121 B. Widrow. R. G. Winter and R. A. Baxter, "Layered Neural Nets for pattern recognition". IEEE Trans. on ASSP. No.?. Ju1y 1988.

1131 M. Stevenson, R. Winter and B. Widrow. "Sensivity of reedforward Neural Networks to weight errors". IEEE Trans. on Neural Network. Vol. 1. No. 1. March IQQO.
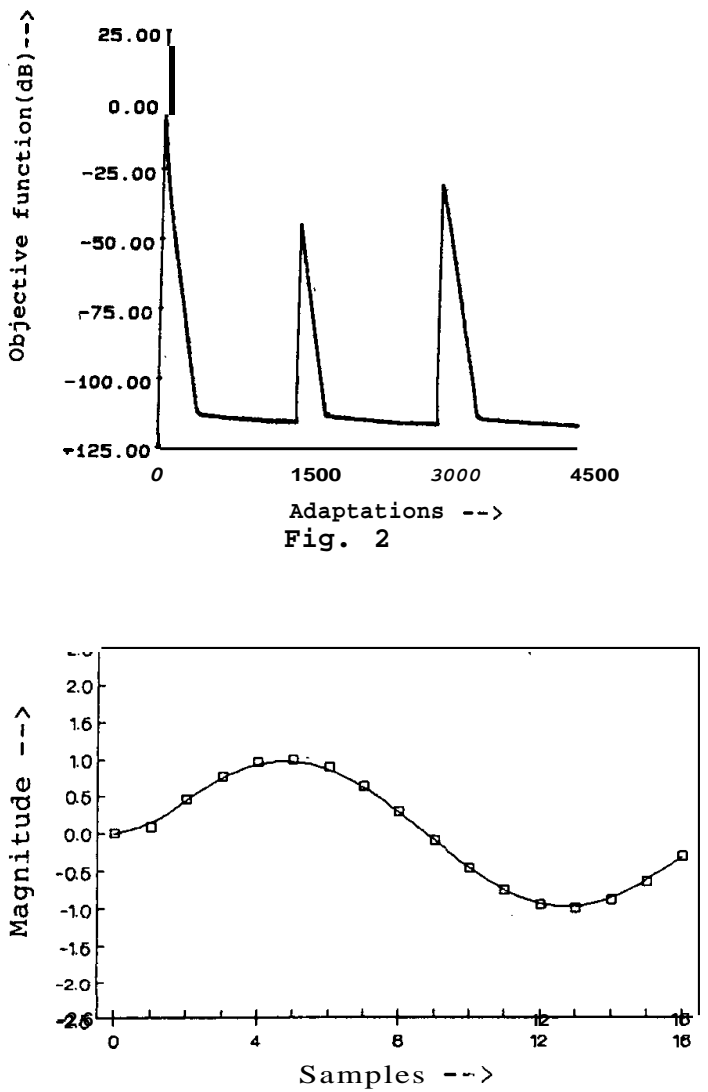

Fig. 3

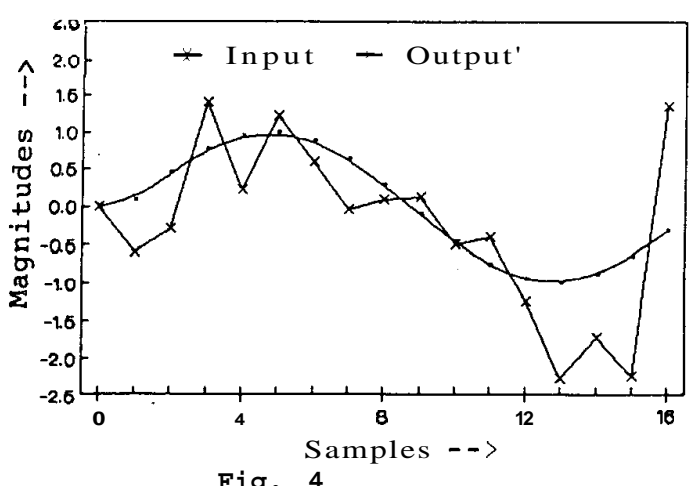

Fig. e: Learning Curve for Fundamental Samples using backpropagation algorithm $\mathrm{C} 3$ sets of training signals), $n=0.45, \lambda=0.0065$ $A=3.0, f_{s}=800 \mathrm{~Hz}$.

Fig. 3: Target waveform for fundamentalCsoHz) Fig. 4: Input signal with SNR=OdB \& the prodicted output for fundamental.

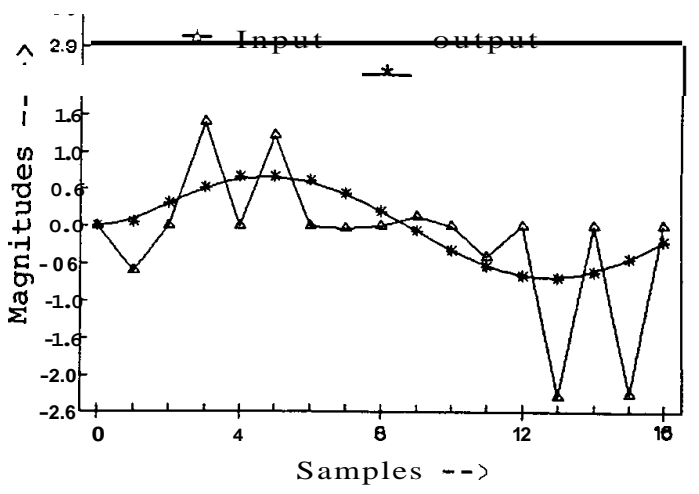

Fig. 5
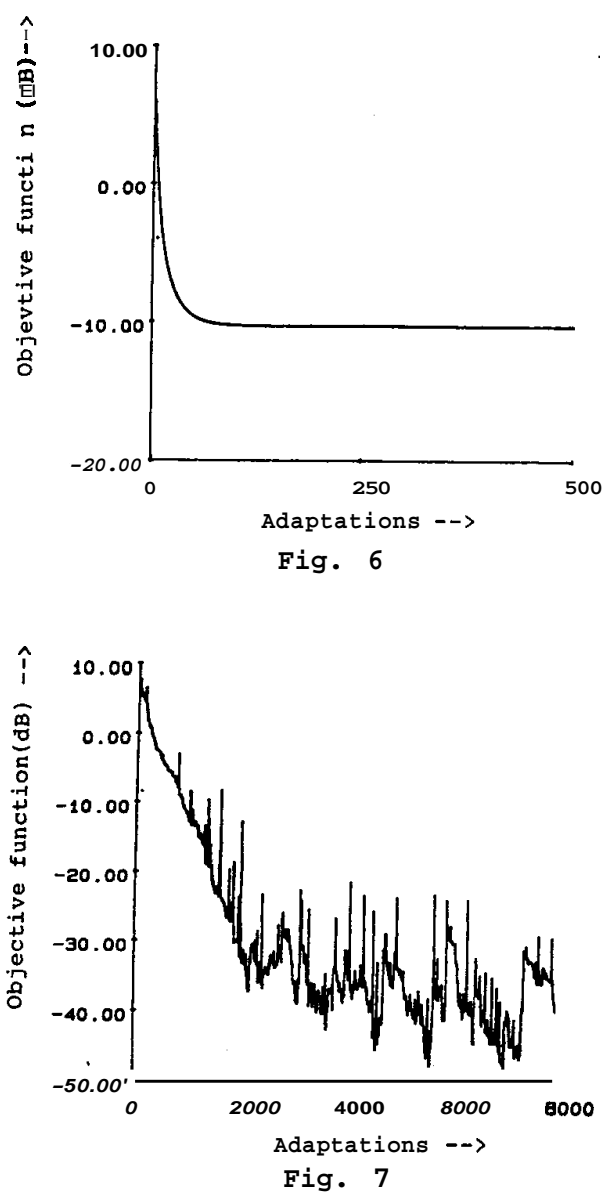

Fig. 5: Input signal. SNR=0dB with samples suppressedC1.e $3,4, \ldots 163$ and the correspondling predicted output for fundamental.

, Fıg. 6 :Learning Curve for 3 rd HarmoniccBack propagation Al. J, $n=0.5, \lambda=0.009, A=2,4$.

Fig. 7:Learning Curve for $3 \mathrm{rd}$ Harmonic using Cauchy's Al $, \infty=0.01, A=0.07 . A=2.0$. 

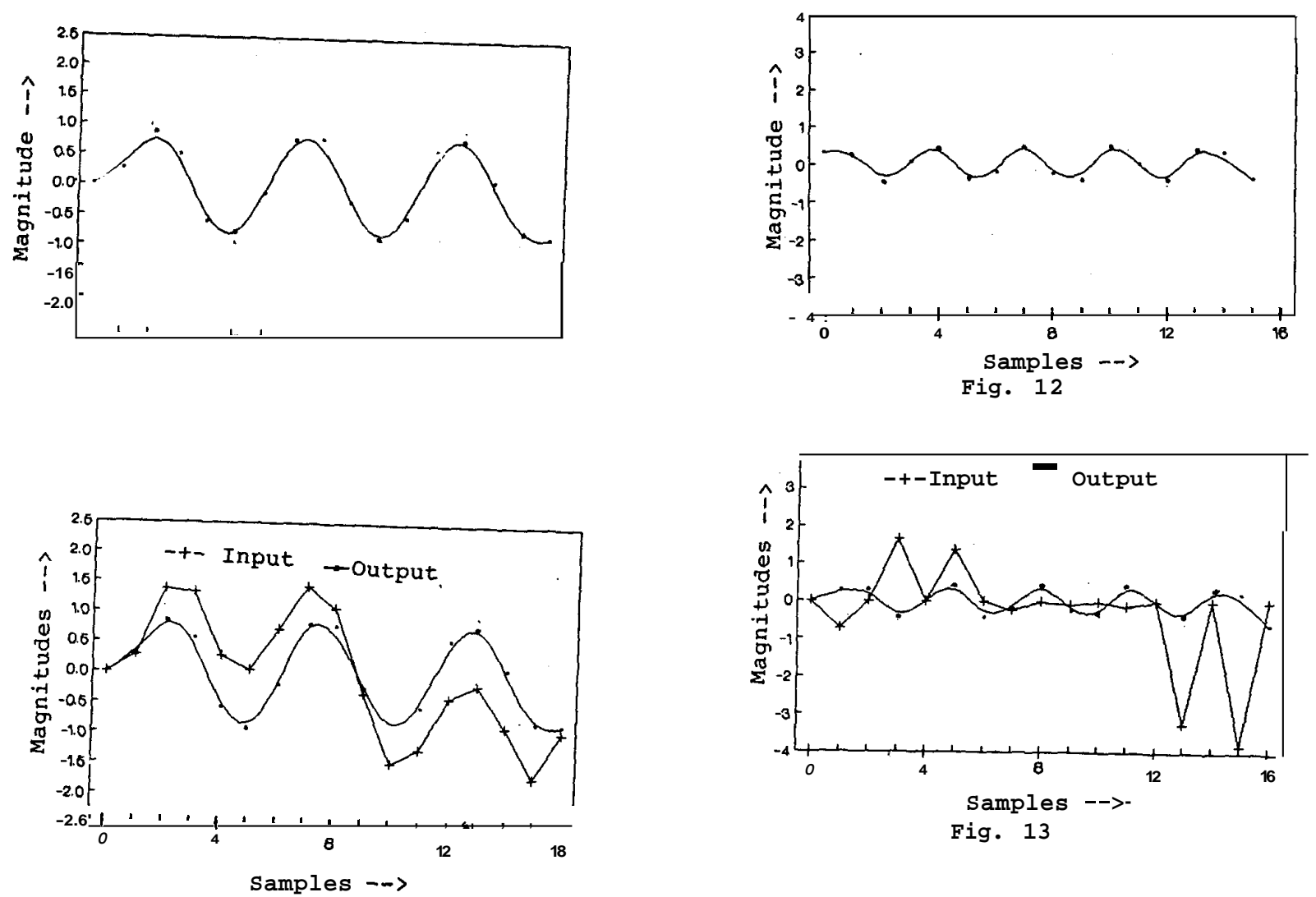

Fig. 13
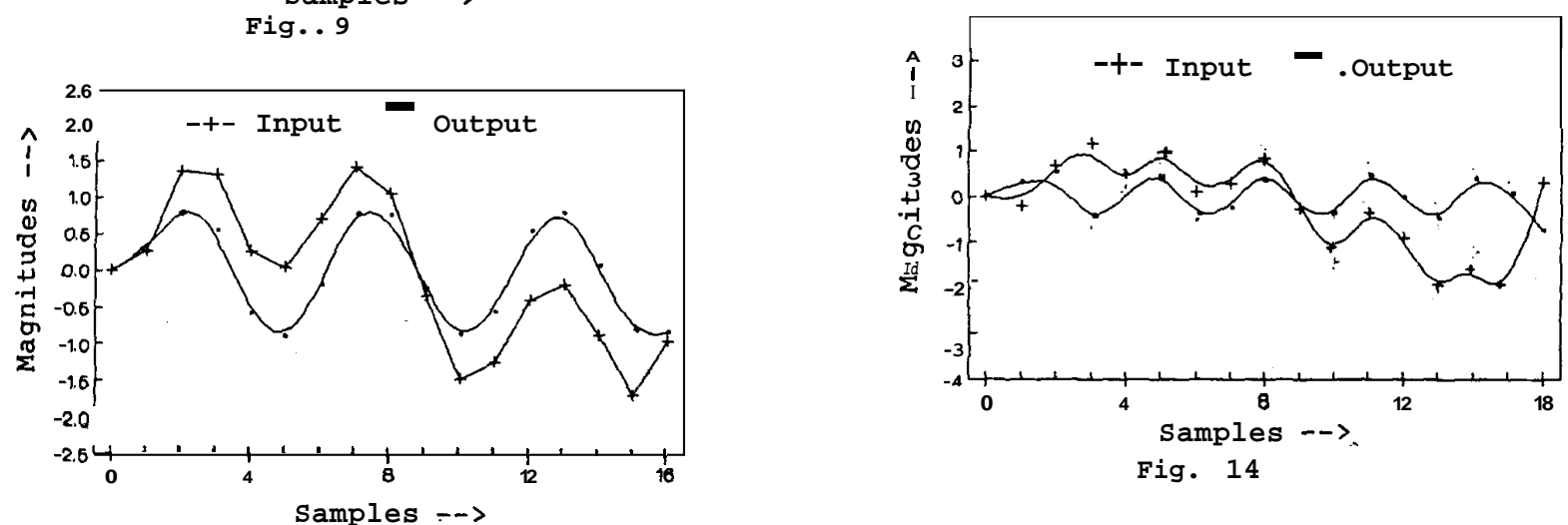

Fig. 10

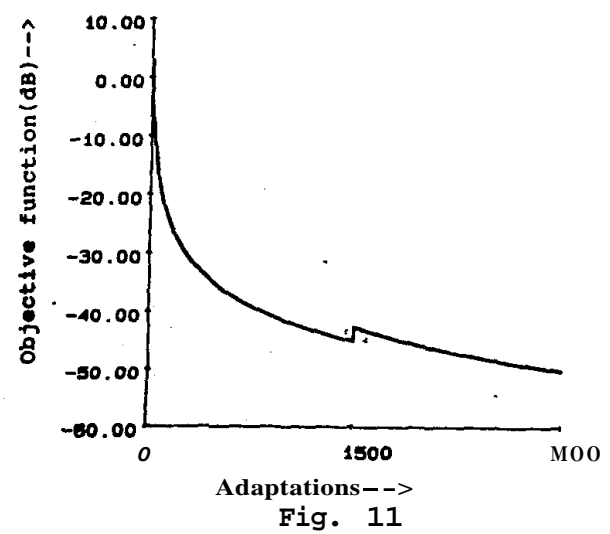

Fig. 8: Target for 3rd Harmonic.

Fig. $9:$ Input signal, $5 N R=-20 d B$ \& the output Cbackpropagation Als

Fig. 10: Input Signal, SNR=-20dB \& output CCauchy*s Algorithm3

Fig. 11: Learing Curve for 5th harmonic Cbackpropagation $\mathrm{Al}$ )

Fig.12 : Target for 5th harmonic.

F1g. 13: Input signal SNR=OdB with tven sample suppressed \& the output.

Fig.14: Input signal $S N R=O d B$ and the output 